\title{
Immunochromatographic detection of MPB64 secreted from active BCG by heating: toward same-day diagnosis of tuberculosis
}

\author{
Kazunari Nakaishi,2, Satoshi Watabe,2, Toshiyuki Kitagawa3 \& Etsuro Ito*,2,4,5
}

\section{ABSTRACT}

We propose a new detection method for a tuberculosis-specific protein, MPB64, obtained from active bacillus CalmetteGuérin (BCG) by heating. When BCG was included in solution at a concentration $>2.75 \times 10^{4} \mathrm{CFU} / \mathrm{ml}$, our method for collecting MPB64 through heating active BCG combined with an immunochromatographic assay detected active bacilli within $2.5 \mathrm{~h}$. By contrast, a culture test, which is the gold standard for tuberculosis diagnosis, does not provide results for between 1 week and 2 months. The rapid tests based on PCR have some drawbacks, for example they detect DNA from both active and latent (or even dead) tubercle bacilli. Therefore, our method may pave the way toward detecting only active tubercle bacilli at a reasonable cost and providing same-day diagnosis.

\section{METHOD SUMMARY}

BCG is heated to secrete a specific Mycobacterium tuberculosis protein, MPB64, which is an indicator of active tubercle bacilli. MPB64 is detected by an immunochromatographic assay using platinum-gold colloid. The time from preparation of specimens to detection of MPB64 (i.e., active tubercle bacilli) is only $2.5 \mathrm{~h}$.

\section{KEYWORDS:}

immunochromatography - MPB64 • Mycobacterium tuberculosis • same-day diagnosis

${ }^{7} R \& D$ Headquarters, TAUNS Laboratories, Inc. 761-1 Kamishima, Izunokuni, Shizuoka 410-2325, Japan; ${ }^{2}$ Waseda Research Institute for Science \& Engineering, Waseda University, 3-4-1 Okubo, Shinjuku, Tokyo 169-8555, Japan; ${ }^{3}$ Quality Control Department, TAUNS Laboratories, Inc., 761-1 Kamishima, Izunokuni, Shizuoka 410-2325, Japan; ${ }^{4}$ Department of Biology, Waseda University, 2-2 Wakamatsucho, Shinjuku, Tokyo 162-8480, Japan; ${ }^{5}$ Graduate Institute of Medicine, Kaohsiung Medical University, 100 Shih-Chuan 1st Rd, Sanmin, Kaohsiung 80756, Taiwan; *Author for correspondence: eito@waseda.jp

BioTechniques 66: 240-242 (May 2019) 10.2144/ btn-2019-0020
Tuberculosis (TB), a disease caused by the bacterium Mycobacterium tuberculosis var. tuberculosis, is one of the top ten causes of death worldwide [1]. The WHO reported that in 2017, 10 million people contracted TB and 1.6 million people died from the disease (including 0.3 million people with HIV) [2]. Approximately a quarter of the world's population has latent TB; that is, infection by tubercle bacilli, but are asymptomatic [2]. Although an estimated 54 million lives were saved through TB diagnosis and treatment between 2000 and 2017 [2], more timely diagnosis and better treatment are required in low- and middle-income countries.

Use of the PCR-based tests, such as Xpert MTB/RIF (Cepheid, CA, USA) and Amplicor Mycobacterium tuberculosis Test (Roche, Basel, Switzerland), has expanded substantially [3]. Diagnosis can be made within $2 \mathrm{~h}$, and these tests are recommended as the initial diagnostic test for all individuals with signs and symptoms of TB [2]. These tests, however, have some critical drawbacks. Whereas the positive ratio in smear-positive specimens is $>90 \%$, that in smear-negative specimens is approximately $70 \%$ [4]; therefore, PCR-based tests also detect latent (or even dead) tubercle bacilli. Moreover, hemoglobin and some factors present after dissolving sputum may retard PCR-based tests, providing pseudo-negative results [5]. Thus, PCR-based tests are not suitable for detecting active tubercle bacilli in patients during treatment, even though Xpert MTB/RIF can detect resistance to rifampicin, the most effective TB medicine [6]. Further, a loop-mediated isothermal amplification (LAMP) assay (Eiken Chemical, Tokyo, Japan) has been proposed as an isothermal molecular method [7]. LAMP offers potential advantages over the PCR-based tests in its simplicity, speed, specificity and cost-effectiveness. Although LAMP is thought to be useful, it also detects both active and latent tubercle bacilli, which is problematic.

In the present study, we attempt to detect only active tubercle bacilli, neither latent nor dead ones, and propose a new rapid method to easily obtain and detect a specific Mycobacterium tuberculosis protein, MPB64 [8], without culture. In our proposed method, when active bacilli are heated they secrete some secretory proteins including MPB64. It has been confirmed that MPB64 is secreted from the following eight strains of $M$. tuberculosis complex: M. tuberculosis H37Rv, M. tuberculosis H37Ra, M. africanum, $M$. bovis deer, $M$. microti, $M$. bovis bacillus Calmette-Guérin (BCG)-Tokyo, M. bovis BCG-Russia, and M. bovis BCG-Moreau [9]. BCG (Mycobacterium tuberculosis var. bovis) is used as tubercle bacilli. MPB64 as an indicator of active BCG is detected through an immunochromatographic assay. Although the detection time in our present immunochromatographic assay combined with a heat protocol is much shorter $(\sim 2.5 \mathrm{~h})$ than a culture method (1 week to 2 months), the detection sensitivity for tubercle bacilli is still poorer than culture tests.

Instead of $M$. tuberculosis var. tuberculosis, the BCG-Tokyo strain of tubercle bacilli was used (a kind gift from a branch of the University of Tokyo Hospital). We prepared a diluted series of BCG in heat treatment buffer comprising $2 \%$ Tween 20 and $50 \mathrm{mM}$ glucose in threefold dilutions of dehydrated culture medium (Difco Middlebrook 7H9 Broth, Cat \#271310, Becton Dickinson, NJ, USA). The concentrations of BCG were estimated according to MacFarland standard No 1.0 in the following immunochromatographic and culture tests. 
- BCG bacteria suspended in $200 \mu \mathrm{l}$ of heat treatment buffer were heated in an aluminum block heater at $46^{\circ} \mathrm{C}$ for $1 \mathrm{~h}$, resulting in the secretion of MPB64. To detect MPB64 protein, we manufactured an immunochromatographic assay based on the Capilia TB-Neo assay (TAUNS, Shizuoka, Japan) [10]. Briefly, the main modification was changing the colloidal particles from gold colloid to platinum-gold colloid. The color of gold colloid is reddish purple, and that of platinum-gold colloid is black. The membrane used in immunochromatography is white. That is, the line formed by a color reaction with platinumgold colloid has high contrast, and thus it can be easily observed not only by visual inspection but also by a measuring device. Therefore, the sensitivity of immunochromatography becomes better when using platinum-gold colloid [11].

The colloidal particles were labeled with an antibody that reacts with the target antigen MPB64 and forms an antigenantibody complex. The antibodies used were the same as those in the Capilia TB-Neo assay [10]. The detection sensitivity was much higher than that of the Capilia TB-Neo assay, whose limit of detection (LOD) is reported to be $1.2 \times 10^{6} \mathrm{CFU} / \mathrm{ml}$ (values for the present method are shown in Table 1) [9]. We placed $80 \mu$ l of the specimen into the test well of an immunochromatographic plate and read the line formed by a color reaction appearing after $15 \mathrm{~min}$. Line detection on each immunochromatographic plate was performed in a blinded manner.
To accurately count the number of BCG, we performed culture tests in addition to immunochromatographic tests. Culture medium powder (19 g, Difco Middlebrook 7H10 Agar, Cat \#262710, Becton Dickinson) was suspended in $900 \mathrm{ml}$ of distilled water containing $5 \mathrm{ml}$ glycerol, then autoclaved. Oleic acid, bovine albumin, sodium chloride, dextrose and catalase $(100 \mathrm{ml}$, for their concentrations see BBL Middlebrook OADC Enrichment, Cat \#211886, Becton Dickinson) were added to the culture medium, which was then poured into Petri dishes for cultures. BCG bacilli in heat treatment buffer $(100 \mu \mathrm{l})$ were coated onto the culture medium in a Petri dish and cultivated at $37^{\circ} \mathrm{C}$ for up to 2 months, and the bacterial colonies were counted.

Using a diluted series of BCG in heat treatment buffer, we performed immunochromatographic tests and culture tests (Table 1). When BCG was included in solution at a concentration $>2.75 \times 10^{4}$ $\mathrm{CFU} / \mathrm{ml}$, our method of collecting MPB64 through heating active BCG combined with the immunochromatographic assay successfully detected active bacilli. We have performed the experiments more than 200 times, and obtained the data consistently. BCG was used at a concentration $>2.75 \times 10^{4}$ CFU/ml; the specificity was $100 \%$ compared with the culture tests. Further, when we performed the control experiments without heating, no color reaction was observed by a chromatographic assay. Although the LOD of the present method was slightly poorer than that of the culture test, which is the gold standard for TB diagnosis (e.g., $<10^{3}$
CFU/ml) [12], our method may pave the way toward detecting only active tubercle bacilli at a reasonable cost and providing same-day diagnosis.

Our present test, similar to the culture test, is able to detect only active tubercle bacilli and can thus be used to identify bacilli and evaluate drug sensitivity. Drug sensitivity can be evaluated by checking whether the drug, which is administered to patients, kills the bacilli, because our proposed technique detects only active bacilli. Furthermore, the most important feature of our method is the short examination time (only $2.5 \mathrm{~h}$ ) from preparation of specimens to detection of tubercle bacilli, including the heating time to secrete MPB64 and the color reaction time in immunochromatography; by contrast, culture tests do not provide results for between 1 week and 2 months.

Smear tests are rapid and easy to use, although their sensitivity is poor [13]. Recently, some other approaches using immunochromatography were reported as a rapid TB diagnosis kit [14]. However, these kits still used culture supernatant. In other words, they needed time including culture. Our proposed method using a heating process is decidedly superior to the rapid TB diagnosis kits proposed so far. IFN- $\gamma$-release assays (IGRAs), including the QFT Plus and T-SPOT, are now recommended by the WHO for detection of latent tubercle bacilli in low- and middle-income countries [15]. However, as previously reported: (1) IGRAs and the tuberculin skin test (TST) cannot accurately predict the risk

\begin{tabular}{|c|c|}
\hline Number of BCG (CFU/ml) counted by culture methods & Visual confirmation by immunochromatographic assays \\
\hline 0 & - \\
\hline $2.75 \times 10^{4}$ & \pm \\
\hline $5.50 \times 10^{4}$ & + \\
\hline $2.20 \times 10^{5}$ & + \\
\hline $4.40 \times 10^{5}$ & + \\
\hline $1.32 \times 10^{6}$ & $2+$ \\
\hline $2.20 \times 10^{6}$ & $2+$ \\
\hline $4.40 \times 10^{6}$ & $2+$ \\
\hline $8.80 \times 10^{6}$ & $2+$ \\
\hline
\end{tabular}


of infected individuals developing active TB disease; (2) neither IGRAs nor the TST should be used for the diagnosis of active TB disease; and (3) IGRAs are more costly and technically complex to perform than the TST. Given the comparable performance but higher cost of IGRAs, replacing the TST with IGRAs as a public health intervention in resource-constrained settings is not recommended.

In the present study, MPB64, which is secreted only from active tubercle bacilli, was detected with an immunochromatographic assay. The LOD, however, requires further improvement. The present method detected approximately $10^{4} \mathrm{CFU} / \mathrm{ml}$, whereas the conventional culture method detected approximately $<10^{3} \mathrm{CFU} / \mathrm{ml}$ [11]. Combining the present method for eliciting MPB64 secretion by heating with a new ultrasensitive ELISA coupled with thionicotinamide-adenine dinucleotide (thio-NAD) cycling [16,17] might enable TB diagnosis as a same-day test with a higher LOD. This assay will be further developed in our future work.

\section{AUTHOR CONTRIBUTIONS}

$\mathrm{KN}$ and SW designed the study. KN, SW and TK collected and analyzed the data. El interpreted and analyzed the data and wrote the manuscript.

\section{FINANCIAL \& COMPETING INTERESTS DISCLOSURE}

KN, SW and TK are employees of TAUNS Laboratories, Inc. The authors have no other relevant affiliations or financial involvement with any organization or entity with a financial interest in or financial conflict with the subject matter or materials discussed in the manuscript apart from those disclosed.

No writing assistance was utilized in the production of this manuscript.

\section{OPEN ACCESS}

This work is licensed under the AttributionNonCommercial-NoDerivatives 4.0 Unported License. To view a copy of this license, visit http://creativecommons.org/licenses/ by-nc-nd/4.0/

\section{REFERENCES}

1. Jeliñsk A, Zajac M, Dadej A, Tomczak S, Geszke-Moritz M, Muszalska I. Tuberculosis - present medication and therapeutic prospects. Curr. Med. Chem. 25, 1 (2018).

2. World Health Organization. Fact Sheets, Tuberculosis. www.who.int/en/news-room/fact-sheets/detail/tuberculosis

3. Rice JP, Seifert M, Moser KS, Rodwell TC. Performance of the Xpert MTB/RIF assay for the diagnosis of pulmonary tuberculosis and rifampin resistance in a low-incidence, high-resource setting. PLOS One 12 e0186139 (2017).

4. Carpentier E, Drouillard B, Dailloux $\mathrm{M}$ et al. Diagnosis of tuberculosis by Amplicor Mycobacterium tuberculosis test: a multicenter study. J. Clin. Microbiol. 33, 3106-3110 (1995).

5. Beavis KG, Lichty MB, Jungkind DL, Giger O. Evaluation of Amplicor PCR for direct detection of Mycobacterium tuberculosis from sputum specimens. J. Clin. Microbio. 33, 2582-2586 (1995).
6. Boyd R, Ford N, Padgen P, Cox H. Time to treatment for rifampicin-resistant tuberculosis: systematic review an meta-analysis. Int. J. Tuberc. Lung Dis. 21, 1173-1180 (2017).

7. Notomi T, Okayama H, Masubuchi $\mathrm{H}$ et al. Loop-mediated isothermal amplification of DNA. Nucleic Acids Res. 28, E63 (2000).

8. Harboe M, Nagai S, Patarroyo ME, Torres ML, Ramirez C, Cruz N. Properties of proteins MPB64, MPB70, and MPB80 of Mycobacterium bovis BCG. Infect. Immun. 52, 293-302 (1986)

9. TAUNS. Capilia TB-Neo. www.tauns.co.jp/english/ product/Capiliatb-Neo_insert.pdf\#search $=\% 27$ capiliar+TB+neo\%27

10. Chikamatsu $\mathrm{K}$, Aono $\mathrm{A}$, Yamada $\mathrm{H}$ et al. Comparative evaluation of three immunochromatographic identification tests for culture confirmation of Mycobacterium tuberculosis complex. BMC Infect. Dis. 14, 54 (2014).

11. Iwatsuki-Horimoto $K$, Shi J, Wang $X$ et al. Development of an influenza rapid diagnostic kit specific for the $\mathrm{H} 7$ subtype. Front. Microbiol. 9, 1346 (2018).

12. Messelhäusser $U$, Kämpf $P$, Hörmansdorfer $S$ et al. Culture and molecular method for detection of Mycobac terium tuberculosis complex and Mycobacterium avium subsp. paratuberculosis in milk and dairy products. Appl. Environ. Microbiol. 78, 295-297 (2012).

13. Gordin F, Slutkin G. The validity of acid-fast smears in the diagnosis of pulmonary tuberculosis. Arch. Pathol. Lab. Med. 114, 1025-1027 (1990).

14. Wu $X$, Wang $Y$, Weng $T$ et al. Preparation of immunochromatographic strips for rapid detection of early secreted protein ESAT-6 and culture filtrate protein CFP-10 from Mycobacterium tuberculosis. Medicine (Baltimore). 96, e9350 (2017).

15. World Health Organization. Tuberculosis. www.who.int/ tb/features_archive/igra_policy24oct/en/

16. Watabe $\mathrm{S}$, Kodama $\mathrm{H}$, Kaneda $\mathrm{M}$ et al. Ultrasensitive enzyme-linked immunosorbent assay (ELISA) of proteins by combination with the thio-NAD cycling method. Biophysics 10, 49-54 (2014)

17. Morikawa M, Naito R, Mita K et al. Subattomole detection of adiponectin in urine by ultrasensitive ELISA coupled with thio-NAD cycling. Biophys. Physicobiol. 12, 79-86 (2015). 